\title{
Abbreviations of editions, commentaries and works of reference
}

\author{
A-B \\ Austin, C. and Bastianini, G. (2002), Posidippi Pellaei quae supersunt omnia, \\ Milan. \\ AnOx Cramer, J. A. (1835-37), Anecdota Graeca e codd. manuscriptis \\ bibliothecarum Oxoniensium, 4 vols, Oxford. \\ $A P$ \\ Anthologia Palatina. \\ $C A$ \\ CEG \\ Powell, J. U. (1925 [reprinted 1970]), Collectanea Alexandrina: reliquiae \\ minores poetarum graecorum aetatis ptolemaicae, 323-146 A.C., epicorum, \\ elegiacorum, lyricorum, ethicorum, Oxford. \\ Hansen, P. A. (1983-89), Carmina Epigraphica Graeca, 2 vols, Berlin-New \\ York. \\ CLGP Commentaria et Lexica Graeca in Papyris Reperta (2003-). I.1.1: Bastianini, \\ G., Haslam, M., Maehler, H., Montanari, F., and Römer, C. (with Stroppa, M.) \\ (2004), Pars I: Commentaria et lexica in auctores; Vol. 1, fasc. 1: Aeschines - \\ Alcaeus, Munich-Leipzig. \\ I.1.2.1: Bastianini, G., Haslam, M., Maehler, H., Montanari, F., and Römer, C., \\ Stroppa, M. (2013), Pars I: Commentaria et lexica in auctores; Vol. 1, \\ fasc. 2.1 Alcman, Berlin-Boston. \\ I.1.3: Bastianini, G., Haslam, M., Maehler, H., Montanari, F., and Römer, C. \\ (with Stroppa, M.) (2011), Pars I: Commentaria et lexica in auctores; Vol. 1, \\ fasc. 3: Apollonius Rhodius - Aristides, Berlin-Boston. \\ I.2.6: Bastianini, G. Colomo, D., Haslam, M., Maehler, H., Montana, F., \\ Montanari, F., and Römer, C. (2019), Pars I: Commentaria et lexica in \\ auctores; Vol. 2, fasc. 6: Galenus - Hipponax, Berlin-Boston. \\ CPF \\ Corpus dei papiri filosofici greci e latini (1989-), Florence. \\ $\mathrm{D}-\mathrm{K}$ \\ Diels, H. (1951-52), Die Fragmente der Vorsokratiker, 3 vols, 6th edn, revised \\ by W. Kranz, Berlin. \\ $E D G$ \\ Beekes, R. S. B. (with L. van Beek) (2010), Etymological Dictionary of Greek \\ (Leiden Indo-European Etymological Dictionary Series Volume 10), 2 vols, \\ Leiden-Boston. \\ EGEF Tsagalis, C. (2017), Early Greek Epic Fragments I: Antiquarian and \\ Genealogical Epic, Trends in Classics (Supplementary Volumes 47), Berlin- \\ Boston. \\ EGF Davies, M. (1988), Epicorum Graecorum Fragmenta, Göttingen. \\ EGM Fowler, R. L. (2013), Early Greek Mythography, 2 vols, Oxford. \\ Et.Gen. Miller, E. (1868), Mélanges de littérature grecque contenant un grand nombre \\ de textes inédits, Paris. \\ Calame, C. (1970), Etymologicum Genuinum. Les citations de poètes lyriques, \\ Rome. \\ Et.Magn. Gaisford, T. (1848), Etymologicum Magnum, Oxford [reprinted in Amsterdam, \\ 1967]. \\ EV Enciclopedia Virgiliana (1984-91), 5 vols, Rome.
}


$\mathrm{F}$

FGE

FGrHist

GCS

GEF

GEW

GG

GL

$G M A W^{2}$

G-P

GVI

$\mathrm{H}$

$H E$

Ho.

$I E G^{2}$

IG XII.7

IK

IMT

L-M

$\angle D A B$

LfgrE

LSJ ${ }^{9}$

M-W

$\mathrm{MP}^{3}$

$O C D^{4}$

[only in Chapter 2] Finglass' edition in Finglass and Davies (see Works cited by author's name)

Page, D. L. (1981), Further Greek Epigrams, revised and prepared for publication by R. D. Dawe and J. Diggle, Cambridge.

Jacoby, F. et al. (1923-), Die Fragmente der griechischen Historiker, LeidenBoston-Cologne.

Markschies, C. (1897-), Die griechischen christlichen Schriftsteller der erstern Jahrhunderte.

West, M. L. (2003), Greek Epic Fragments from the Seventh to the Fifth Centuries BC, Cambridge, MA-London.

Frisk, J. I. H. (1954-70), Griechisches etymologisches Wörterbuch, 2 vols, Heidelberg.

Uhlig, G. et al. (1965), Grammatici Graeci, 4 vols, Hildesheim.

Keil, G. T. H. (1857-80), Grammatici Latini, 8 vols, with supplement by H. Hagen, Leipzig.

Turner, E. G. (1987²), Greek Manuscripts of the Ancient World, 2nd edn revised and enlarged by P. J. Parsons (BICS Supplement 46), London.

Gentili, B. and Prato, C. (1988-2002), Poetarum Elegiacorum testimonia et fragmenta, 2nd end, 2 vols, Leipzig.

Peek, W. (1955), Griechische Vers-Inschriften, Amsterdam.

[only in Chapter 13] Hirschberger (see Works cited by author's name)

Gow, A. S. F. and Page, D. L. (1965), The Greek Anthology. Hellenistic Epigrams, 2 vols, Cambridge.

[only in Chapter 3] Hollis (see Works cited by author's name)

West, M. L. (1989-92 [original edn 1971-72]), lambi et elegi Graeci ante Alexandrum cantati, 2 vols, 2nd edn, Oxford.

Delamarre, J. (1908), Inscriptiones Graecae XII.7. Inscriptiones Amorgi et insularum vicinarum, Berlin.

Inschriften griechischer Städte aus Kleinasien (1972-).

Barth, M. and Stauber, J. (1996), Inschriften Mysia \& Troas, Munich.

Laks, A. and Most, G. W. (2016), Early Greek Philosophy, 9 vols, Cambridge, MA.

Leuven Database of Ancient Books

(https://www.trismegistos.org/ldab/)

Snell, B. et. al. (1955-2010), Lexikon des frühgriechischen Epos, 25 vols, Göttingen.

Liddell, H. G. and Scott, R. (1940), A Greek-English Lexicon, $9^{\text {th }}$ edn, revised by $\mathrm{H}$. Stuart-Jones et al., Oxford.

Merkelbach, R. and West M. L. (1967), Fragmenta Hesiodea. Oxford (ed. maior).

Mertens-Pack ${ }^{3}$ Online Database (version of March 2014).

http://promethee.philo.ulg.ac.be/cedopal/index.htm).

Hornblower, S. and Spawforth, A. (2012), Oxford Classical Dictionary, 4th edn, Oxford. 
$\mathrm{P}$

$P C G$

PEG

PLF

$P M G$

PMGF

PPF

$R E$

Roscher, Lex. $\quad$ Roscher, W. H. (1884-1937), Ausführliches Lexikon d. griechischen u. römischen Mythologie, 6 vols in 9, Leipzig.

SEG

SGO

SH

$S L G$

S-M

Suda

TLG

TM

$\operatorname{TrGF}$

[only in Chapter 7] Poltera (see Works cited by author's name) New York. 1987]. edn 1962]. Alcman, Stesichorus, Ibycus, Oxford.

Diels, H. (1901), Poetarum Philosophorum Fragmenta, Berlin. Georg Wissowa unter Mitwirkung zahlreicher Fachgenossen, 83 vols, Supplementum Epigraphicum Graecum (Leiden 1923-71; Amsterdam 1971-). griechischen Osten, 5 vols, Stuttgart. Berlin-New York.

Page, D. L. (1974), Supplementum Lyricis Graecis. Poetarum lyricorum Graecorum fragmenta quae recens innotuerunt, Oxford. Leipzig.

Adler, A. (1928-38), Suidae Lexicon, 5 vols, Leipzig.

Thesaurus Linguae Graecae

Trismegistos (https://www.trismegistos.org/index.php)
Kassel, R. and Austin, C. F. L. (1983-), Poetae Comici Graeci, 8 vols, Berlin-

Bernabé, A. (1996-2007), Poetae Epici Graeci. Testimonia e Fragmenta, 2nd edn, 2 Parts, Stuttgart-Munich-Leipzig-Berlin-New York [1st edn of vol.1

Lobel, E. and Page, D. (1955), Poetarum Lesbiorum Fragmenta, Oxford. Page, D. L. (1967), Poetae Melici Graeci [corrected reprint], Oxford [original

Davies, M. (1991-), Poetarum Melicorum Graecorum Fragmenta, vol. 1:

Pauly, A. F. von, Wissowa, G., Kroll, W. et al. (1894-1980), Real-Encyclopädie der klassischen Altertumswissenschaft. Neue Bearbeitung begonnen von

Merkelbach, R. and Stauber, J. (1998-2004), Steinepigramme aus dem

Lloyd-Jones, H. and Parsons, P. J. (1983), Supplementum Hellenisticum,

Snell, B. and Maehler, H. (1987-9), Pindari Carmina cum Fragmentis, 2 vols,

Snell, B. et al. (1971-2004), Tragicorum Graecorum Fragmenta, 5 vols, Göttingen.

I: Didascaliae Tragicae, Catalogi Tragicorum et Tragoediarum, Testimonia et Fragmenta Tragicorum Minorum (B. Snell; 2nd edn revised by R. Kannicht; Göttingen $1971^{1}$ and $1986^{2}$ ).

II: Fragmenta Adespota (R. Kannicht and B. Snell; Göttingen 1981).

III: Aeschylus (S. L. Radt; Göttingen 1985).

IV: Sophocles (S. L. Radt; Göttingen $1977^{1}$ and $1999^{2}$ ).

V: Euripides (R. Kannicht; 2 parts; Göttingen 2004). 
